\title{
Assessing the relationship between water and nutrition knowledge and beverage consumption habits in children
}

\author{
Bridget R Irwin 1,2, Mark R Speechley ${ }^{2,3}$ and Jason A Gilliland 1,2,4,5,6,7,8,* \\ 'Human Environments Analysis Laboratory, Western University, Social Science Centre, 1151 Richmond Street, \\ London, Ontario, Canada, N6A 5C2: '2Department of Epidemiology and Biostatistics, Western University, London, \\ Ontario, Canada: ${ }^{3}$ Schulich Interfaculty Program in Public Health, Western University, London, Ontario, Canada: \\ ${ }^{4}$ Department of Paediatrics, Western University, London, Ontario, Canada: ${ }^{5}$ Department of Geography, Western \\ University, London, Ontario, Canada: 'SSchool of Health Studies, Western University, London, Ontario, Canada: \\ ${ }^{7}$ Children's Health Research Institute, London, Ontario, Canada: ${ }^{~}$ Lawson Health Research Institute, London, Ontario, \\ Canada
}

Submitted 14 September 2018: Final revision received 31 December 2018: Accepted 8 February 2019; First published online 14 May 2019

\begin{abstract}
Objective: To examine the relationship between knowledge and beverage consumption habits among children.

Design: Cross-sectional analysis. Linear regression was used to identify sociodemographic, dietary and behavioural determinants of beverage consumption and knowledge, and to describe the relationships between children's knowledge and water and sugar-sweetened beverage (SSB) consumption.

Settings: Seventeen elementary schools in London, Ontario, Canada.

Participants: A total of 1049 children aged 8-14 years.

Results: Knowledge scores were low overall. Children with higher knowledge scores consumed significantly fewer SSB $(\beta=-0.33 ; 95 \%$ CI $-0 \cdot 49,-0 \cdot 18 ; P<0 \cdot 0001)$ and significantly more water $(\beta=0.34 ; 95 \%$ CI $0.16,0.52 ; P=0.0002)$. More frequent refillable water bottle use, lower junk food consumption, lower fruit and vegetable consumption, female sex, higher parental education, two-parent households and not participating in a milk programme were associated with a higher water consumption. Male sex, higher junk food consumption, single-parent households, lower parental education, participating in a milk programme, less frequent refillable water bottle use and permission to leave school grounds at lunchtime were associated with a higher SSB consumption. Water was the most frequently consumed beverage; however, $79 \%$ of respondents reported consuming an SSB at least once daily and $50 \%$ reported consuming an SSB three or more times daily.

Conclusions: Elementary-school children have relatively low nutrition and water knowledge and consume high proportions of SSB. Higher knowledge is associated with increased water consumption and reduced SSB consumption. Interventions to increase knowledge may be effective at improving children's beverage consumption habits.
\end{abstract}

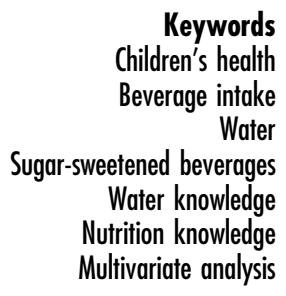

Childhood obesity has emerged as a major public health concern of the 21st century. In Canada alone, the prevalence of overweight and obesity has doubled in the past 40 years, now affecting roughly $30 \%$ of children aged $5-17$ years $^{(1,2)}$. There is strong evidence to suggest that overweight and obese children are at an increased risk of a number of non-communicable diseases and socio-psychological issues including CVD, type 2 diabetes, hypertension and depres$\operatorname{sion}^{(3)}$. These complications follow a child throughout his/ her life course, affecting adult morbidity and mortality ${ }^{(4)}$.
Children's beverage consumption habits have been linked to the rising levels of overweight and obesity observed globally ${ }^{(5-10)}$. According to the Academy of Nutrition and Dietetics, children require approximately 6 to 8 cups of water in total each day to maintain sufficient hydration ${ }^{(11)}$ and healthy body weight ${ }^{(12)}$. Nationwide survey data, however, suggest that few American children are consuming enough water ${ }^{(13,14)}$. Similarly, findings from the Canadian Community Health Survey (CCHS) demonstrate that just $60 \%$ of children's beverage intake consists of healthy drinks 
such as water, milk and $100 \%$ fruit juice, with sugar-sweetened beverages (SSB), including pop, fruit-flavoured drinks, sports drinks and energy drinks, thought to make up the majority of the difference ${ }^{(15)}$. These beverages account for approximately $44 \%$ of daily sugar intake for Canadian children and adolescents, contributing a substantial proportion of daily energy ${ }^{(1,16)}$, and there is moderate-quality evidence linking their consumption to excess weight gain in children ${ }^{(5,6,8,9,17,18)}$. Given these findings, it is not surprising that SSB consumption has been identified as a key risk factor for being overweight or obese ${ }^{(7)}$, and recent systematic reviews suggest that reducing SSB intake in young children and/or replacing SSB with water, an energy- and sugar-free alternative, is an effective strategy for reducing obesity ${ }^{(6,10)}$.

Most water and SSB interventions can be categorized into three types: (i) health/nutrition education programmes, such as educating children about the adverse health effects of consuming SSB; (ii) environmental changes to discourage SSB or encourage water consumption, such as installing water fountains or removing soft drinks from vending machines; and (iii) policies relating to nutrition, such as banning the provision of SSB at school events. Although these approaches are often used in combination, a review of the published literature reveals that education programmes are the most common, particularly when targeting child populations ${ }^{(19)}$. Education interventions aim to positively influence behaviour through improving knowledge: it is believed that by providing children with the information and skills they require to make healthy and balanced food choices, they will automatically begin to incorporate this into their everyday lives, fostering healthy habits ${ }^{(20-33)}$. It is particularly important to establish healthy dietary habits in childhood, as behaviours formed during this period tend to persist into adulthood and thereby affect long-term health status ${ }^{(34-37)}$.

While an association between health-related knowledge and behaviour has been demonstrated in other contexts, there is mixed evidence concerning the effectiveness of nutrition education programmes in altering dietary habits. A number of studies have assessed the relationship between nutrition knowledge and dietary intake, with the majority finding a weak positive association ${ }^{(38)}$; however, only a few have focused on child populations $^{(39-44)}$ and just one of these included beverages ${ }^{(40)}$. That study identified a negative association between children's nutrition knowledge and sugary drink consumption; however, it was conducted in a restrictive sample of children and adolescents living in rural Sicily and thus may not be generalizable to other populations ${ }^{(40)}$.

Given these gaps, the purpose of the present study was to describe the beverage consumption habits and knowledge of elementary-school children aged 8-14 years in London, Ontario, Canada, and to assess whether greater knowledge is associated with healthier beverage habits. The results will inform the design of future SSB and water education-based interventions targeted towards children.

\section{Methods}

\section{Setting and participants}

The Water Does Wonders project occurred in the city of London, Ontario, Canada, throughout the 2016/17 school year and targeted children in thirteen priority neighbourhoods, as identified by a community needs assessment conducted through the Child and Youth Network ${ }^{(45)}$. Compared with the city of London as a whole, priority neighbourhoods were more likely to contain households that had lower incomes, lower levels of educational attainment and were headed by single parents ${ }^{(45)}$. Of the seventy-eight elementary schools in these neighbourhoods, nineteen were invited and agreed to participate in the study; of these, seventeen were included in the data collection. Data were collected from children in grades 4-8, who were approximately 8 to 14 years old. All children were required to have written parental consent, as well as provide personal assent in order to participate.

\section{Data collection and tools}

Data collection for the present study took place in OctoberNovember 2016. Teams of research assistants, volunteers and graduate students from the Human Environments Analysis Laboratory at Western University administered surveys to participating students. The research teams provided verbal instructions and while they were available to answer questions related to comprehension, spelling and process throughout the survey period, they did not prompt students in any way. Students were provided with a complimentary colour-changing pencil upon completing the survey. Students who were absent on the day of the survey were not given the opportunity to complete the survey at a later date.

The youth survey consisted of ninety-one items under five domains including demographics, beverage consumption habits, food and beverage consumption frequencies, eating and drinking during the school day, and nutrition and water knowledge. Response options included multiple-choice, yes/no, Likert-scale and fill-in-the-blank questions. A parental/guardian version of the survey consisting of fifty items under four domains, measuring basic demographics, the child's eating and drinking habits, and eating and drinking during the school day, was used to supplement information collected from the youth survey.

\section{Outcome measures}

Dietary habits were assessed using an FFQ adapted from a survey developed for a previous study, described elsewhere $^{(46)}$.

\section{Water consumption}

Water consumption was measured as the number of times per day a child reported consuming water, with response categories ranging from 0 to $7+$. In order to account for 
potential differences in children's interpretation of 'times per day', for analysis water consumption frequency was divided by the total beverage consumption frequency reported by each child to obtain standardized proportions. For example, if a child reported consuming water 6 times/d and his/her total beverage intake frequently was 18 instances, $33.3 \%$ of the child's total daily beverage consumption would be attributable to water.

\section{Sugar-sweetened beverage consumption}

SSB consumption was an aggregate variable, derived from summing the number of times per day a child reported consuming regular pop, fruit-flavoured drinks (including sports drinks), energy drinks and chocolate milk, each ranging from 0 to $7+$, for a total SSB frequency possible range of 0 to $28+$. Although there is some debate as to whether sweetened milks and milk alternatives should be considered SSB due to the high number of essential nutrients they contain, we included them in our analysis based on a report published by the Dietitians of Canada ${ }^{(47)}$ and using the definition of SSB defined by the Centers for Disease Control and Prevention ${ }^{(48)}$. Conversely, while sweetened coffee and tea drinks are considered SSB under the Centers for Disease Control and Prevention's definition, they were not included in the current analysis because the nature of the survey did not allow respondents to specify whether or not the tea and coffee beverages they consumed contained added sugar. Additionally, despite the fact that $100 \%$ fruit juices are high in sugar and have a similar effect to SSB on the body ${ }^{(49)}$, they were excluded from the analysis because their sugar is naturally occurring. Diet beverages were also excluded. As with water consumption, for analysis SSB consumption frequency was divided by total beverage consumption frequency to generate the percentage of overall reported beverage consumption frequency attributable to SSB.

\section{Water and nutrition knowledge}

Nutrition knowledge was measured by summing the scores of thirty-six individual questions assessing children's knowledge of the sugar, caffeine and water contents of various foods and beverages. Examples include: 'From the list below, choose the beverages that are high in sugar and/or high in caffeine' (100\% apple juice, black/green tea, cappuccino, chocolate milk, Coca-Cola, coffee, fruit punch, Nestea iced tea, peach drink, Red Bull energy drink, Rock Star energy drink, Sprite, SunnyD, water, white milk); and 'Check the fruit or vegetable that has the most water from each pairing' (cucumber or carrots, apple or peach, carrots or tomatoes, cucumber or beans, strawberries or pears, spinach or corn). The minimum possible score a child could achieve was 0 and the maximum was 36 .

Water knowledge was measured by summing the scores of eight individual questions assessing children's knowledge of the water treatment system, the health benefits of water and the world's water. Examples include:
'Where does the water from your tap come from?' (ground water; Lake Ontario \& Erie; Lake Huron \& Erie; Thames River; I don't know); 'How much water do we need to drink each day?' (3-4 cups, 5-6 cups, 7-8 cups, 9-10 cups); 'Can you name one way that your tap water is treated?'; 'Can you list two ways to conserve water at home or at school?'; 'True or false, bottled water is better than tap water'; 'True or false, water is an unlimited resource'; and 'True or false, we have enough water in Canada for everyone, forever'. The minimum possible score a child could achieve was 0 and the maximum was 8 .

Total knowledge was determined by summing the scores of the nutrition and water knowledge subscales. The minimum score that could be achieved was 0 and the maximum was 44 . For analysis purposes, knowledge scores were treated as continuous.

\section{Other covariates}

Sociodemographic characteristics, including sex (male/ female), age, race (white/non-white), living arrangement (single-parent household/two-parent household/other), parent education (high school or less/college or university/graduate school), parent employment status (employed/unemployed) and household income level (low/low-middle/high-middle/high), were determined using self-administered surveys completed by both the parent and child. Income level classifications were made based on reported annual household income and number of people in the household using methods described in the CCHS Derived Variable Specifications ${ }^{(50)}$. Parentreported data were used wherever possible, due to increased likelihood of accuracy; however, where missing, child-reported data were substituted.

Dietary intake, including daily servings of fruits and vegetables and weekly junk food consumption frequency, was assessed using the FFQ component of the youth survey. Junk food consumption was an aggregate variable, derived from summing the number of times per week a child reported consuming sweetened breakfast cereal, cake/pie/doughnuts, potato chips, chocolate bars, pizza, French fries, hot dogs, ice cream, candy, granola bars and cookies, each ranging from 0 to $6+$, for a possible total junk food frequency range of 0 to $66+$. Information on drinking habits such as frequency of use of a refillable water bottle (never/rarely/sometimes/ usually/always), milk programme participation (yes/no) and permission to leave school grounds at lunchtime (yes/ no) was also collected using the youth survey.

\section{Data analysis}

Data cleaning was performed using the statistical software package IBM SPSS Statistics version 24, while all other statistical analyses were conducted using SAS version 9.4 .

Written parental consent was obtained for 1504 (36.8\%) of 4086 eligible children, of whom 1099 provided assent, were present on the day of the study and thus completed 
the youth survey. One school ( $n$ 26) withdrew from the study following data collection and was thus excluded from the present analysis, resulting in a final sample of sixteen schools. Among the remaining study participants, twentyfour children did not have a corresponding parent survey and were excluded from the analysis due to a lack of sociodemographic information. Wilcoxon Mann-Whitney tests, Fisher's exact tests and Monte Carlo estimation simulations with 40000 simulations revealed no significant differences between children with a parent survey and children without, however, indicating that selection bias on this variable is unlikely. The final number of analysed participants was 1049 parent-child dyads, representing $25.67 \%$ of eligible children and $85.08 \%$ of children who completed surveys.

Fully conditional specification multiple imputation with arbitrary missing data patterns was performed to impute values using SAS version 9.4. Fifty imputed data sets were created, based on the guideline that the number of imputations should be approximately equal to the percentage of incomplete cases ${ }^{(51,52)}$. In our sample, just $58.7 \%$ of participants had complete data for all variables of interest and missing data among the imputed variables ranged from $0.5 \%$ (total knowledge score) to $27.6 \%$ (father's employment status). Approximately $33.9 \%$ of participants were missing data on household income level; however, this variable was not imputed due to the high probability that it was not missing at random. Variables included in the imputation model were all of those included in the final analysis, as well as a number of auxiliary variables that were correlated with or predicted missing variables.

Descriptive statistics including means and frequencies were used to describe the characteristics of the sample, as well as participants' beverage consumption habits and water and nutrition knowledge. Hierarchical multivariable regression models with generalized estimating equations to account for clustering at the school level were used to assess the relationship between knowledge and water and SSB consumption, controlling for potential confounders, and to determine which variables were most predictive of knowledge and water and SSB consumption frequency. Intra-cluster correlation coefficients ranged from 0.04 (95\% CI $0.00,0.09)$ for water consumption to 0.06 (95\% CI $0.002,0.17$ ) for SSB consumption, to 0.09 (95\% CI 0.01 , $0 \cdot 17$ ) for total knowledge score, indicating minimal correlation within schools.

Model 1 adjusted for sociodemographic characteristics including sex, age, ethnicity, household income level, maximum household education, child living arrangement and parental employment status. Model 2 added the dietary variables unhealthy food consumption frequency and daily servings of fruits and vegetables. Model 3 added behavioural factors including frequency of refillable water bottle use, participation in a school milk programme and permission to leave school grounds at lunchtime. These variables were selected based on the literature as well as theoretical plausibility and are hypothesized to affect children's water and/
Table 1 Demographics of the sample of elementary-school children aged 8-14 years and their parents/guardians ( $n$ 1049), London, Ontario, Canada, October-November 2016

\begin{tabular}{|c|c|c|c|}
\hline Characteristic & Mean or $n$ & $\%$ & SD \\
\hline Age (years), mean and SD & $10 \cdot 56$ & - & 1.39 \\
\hline \multicolumn{4}{|l|}{ Grade, $n$ and \% } \\
\hline 4 & 227 & 23.02 & \\
\hline 5 & 240 & $24 \cdot 34$ & \\
\hline 6 & 194 & $19 \cdot 68$ & \\
\hline 7 & 189 & $19 \cdot 17$ & \\
\hline 8 & 136 & $13 \cdot 79$ & \\
\hline \multicolumn{4}{|l|}{ Sex, $n$ and $\%$} \\
\hline Male & 449 & $43 \cdot 26$ & \\
\hline Female & 589 & $56 \cdot 74$ & \\
\hline \multicolumn{4}{|l|}{ Race/ethnicity, $n$ and $\%$} \\
\hline White/Caucasian & 658 & $62 \cdot 73$ & \\
\hline Visible minority/mixed race & 391 & $37 \cdot 27$ & \\
\hline \multicolumn{4}{|l|}{ Household income level, $n$ and $\%$} \\
\hline Low & 85 & $8 \cdot 10$ & \\
\hline Low-middle & 121 & 11.53 & \\
\hline High-middle & 148 & $14 \cdot 11$ & \\
\hline High & 339 & $32 \cdot 32$ & \\
\hline Missing & 356 & 33.94 & \\
\hline \multicolumn{4}{|c|}{ Maximum household education, $n$ and $\%$} \\
\hline High school or less & 121 & 11.89 & \\
\hline College/university & 720 & $70 \cdot 73$ & \\
\hline Graduate school & 177 & 17.39 & \\
\hline \multicolumn{4}{|l|}{ Mother's employment status, $n$ and \% } \\
\hline Employed & 700 & 74.39 & \\
\hline Unemployed & 241 & $25 \cdot 61$ & \\
\hline \multicolumn{4}{|l|}{ Father's employment status, $n$ and $\%$} \\
\hline Employed & 711 & 93.55 & \\
\hline Unemployed & 49 & 6.45 & \\
\hline \multicolumn{4}{|l|}{ Child living arrangement, $n$ and $\%$} \\
\hline Single-parent/guardian household & 218 & 21.06 & \\
\hline Two-parent/guardian household & 813 & 78.55 & \\
\hline Other arrangement & 4 & 0.39 & \\
\hline
\end{tabular}

or SSB consumption. Unadjusted and adjusted results are presented. $P$ values of $<0.05$ were considered statistically significant. Sensitivity analyses were performed using only non-imputed data and using absolute beverage consumption frequencies rather than proportions. No collinearity between covariates was identified.

\section{Results}

\section{Characteristics of the sample}

Sample demographics, dietary habits and nutrition/water knowledge are presented in Tables 1-3. The mean age of respondents was 10.6 (SD 1.4) years, and $56.7 \%$ were female. The majority of participants were Caucasian $(62.7 \%)$, lived in two-parent households (78.6\%) and had college/universityeducated parents (88.1\%). Of mothers and fathers, 74.4 and $93.6 \%$, respectively, were employed, and $32.3 \%$ of households were classified as high income, compared with $8.1 \%$ classified as low income.

\section{Beverage consumption and dietary intake}

Participants self-reported consuming water a mean of $5 \cdot 1$ (SD 1.9) times/d, accounting for approximately 39.8 
Table 2 Dietary behaviours of the sample of elementary-school children aged 8-14 years ( $n$ 1049), London, Ontario, Canada, OctoberNovember 2016

\begin{tabular}{|c|c|c|c|}
\hline Characteristic & $n$ or mean & $\%$ & SD \\
\hline \multicolumn{4}{|l|}{ Type of water consumed at home, $n$ and $\%$} \\
\hline Tap & 395 & 37.65 & \\
\hline Barrel & 42 & 4.00 & \\
\hline Bottle & 268 & 25.55 & \\
\hline Filtered & 434 & 41.37 & \\
\hline \multicolumn{4}{|l|}{ Use of a refillable water bottle, $n$ and $\%$} \\
\hline Yes & 722 & 91.51 & \\
\hline No & 67 & 8.49 & \\
\hline \multicolumn{4}{|l|}{ Frequency of refillable water bottle use, $n$ and $\%$} \\
\hline Never & 31 & 3.06 & \\
\hline Rarely & 70 & 6.92 & \\
\hline Sometimes & 239 & 23.62 & \\
\hline Usually & 337 & 33.30 & \\
\hline Always & 335 & $33 \cdot 10$ & \\
\hline \multicolumn{4}{|l|}{ Family use of a refillable water bottle, $n$ and $\%$} \\
\hline Yes & 865 & 83.66 & \\
\hline No & 169 & $16 \cdot 34$ & \\
\hline \multicolumn{4}{|l|}{ Use of a refillable water bottle at school, $n$ and $\%$} \\
\hline Yes & 870 & 84.80 & \\
\hline No & 156 & $15 \cdot 20$ & \\
\hline \multicolumn{4}{|l|}{ Type of beverage consumed during PA, $n$ and $\%$} \\
\hline Water & 908 & 86.56 & \\
\hline $100 \%$ Juice & 57 & 5.43 & \\
\hline Energy drinks & 20 & 1.91 & \\
\hline Sports drinks & 175 & $16 \cdot 68$ & \\
\hline Other & 21 & 2.00 & \\
\hline Daily servings of fruits and vegetables, mean and SD & 4.39 & - & 2.00 \\
\hline Times junk food consumed/week, mean and SD & $15 \cdot 52$ & - & 10.06 \\
\hline \multicolumn{4}{|l|}{ Times beverages consumed/d, mean and SD } \\
\hline Water & $5 \cdot 13$ & - & 1.93 \\
\hline SSB & 3.92 & - & 4.31 \\
\hline $100 \%$ Juice & 1.86 & - & 1.84 \\
\hline White milk & 2.90 & - & 2.43 \\
\hline Diet pop & 0.37 & - & 0.99 \\
\hline Coffee & 0.25 & - & 0.86 \\
\hline Tea & 0.98 & - & 1.66 \\
\hline Percentage of total daily beverage consumption attributable to water, mean and SD & $39 \cdot 77$ & - & $19 \cdot 61$ \\
\hline Percentage of total daily beverage consumption attributable to SSB, mean and SD & $22 \cdot 03$ & - & $17 \cdot 11$ \\
\hline \multicolumn{4}{|l|}{ Beverages allowed to bring to school, $n$ and \% } \\
\hline Water & 978 & 93.23 & \\
\hline Juice & 472 & 45.00 & \\
\hline Fruit-flavoured drinks & 276 & $26 \cdot 31$ & \\
\hline Milk & 332 & 31.65 & \\
\hline Pop & 145 & $13 \cdot 82$ & \\
\hline \multicolumn{4}{|l|}{ Participation in school milk programme, $n$ and $\%$} \\
\hline Yes & 149 & 14.45 & \\
\hline No/do not have & 882 & 85.55 & \\
\hline \multicolumn{4}{|l|}{ Allowed to leave school grounds at lunchtime, $n$ and $\%$} \\
\hline Yes & 149 & 18.33 & \\
\hline No & 842 & 81.67 & \\
\hline
\end{tabular}

PA, physical activity; SSB, sugar-sweetened beverage.

(SD 19.6) \% of their total daily beverage intake, and SSB a mean of 3.9 (SD 4.3) times/d, accounting for approximately 22.0 (SD 17.1) \% of their total daily beverage intake. About four out of five (79.4\%) children reported consuming an SSB at least once daily, and half (49.6\%) reported consuming an SSB three or more times daily. Comparatively, $98.3 \%$ of students reported consuming water at least once daily, and $86.4 \%$ reported consuming water three or more times daily. On average, participants consumed 4.4 (SD 2.0) servings of fruits and vegetables/d, with approximately $29.6 \%$ meeting Canada's Food Guide recommendation of six or more servings daily. Junk foods were consumed a mean of $15 \cdot 5$ (SD 10.1) times/week, or about $2 \cdot 2$ times/d.

Over one-third (37.7\%) of children reported consuming tap water at home, and $91.5 \%$ reported using a refillable water bottle in their everyday life. Additionally, $84.8 \%$ used a refillable water bottle at school, and $83.7 \%$ said their family members used refillable water bottles. Water was the most frequently consumed beverage during physical activity, selected by $86.6 \%$ of respondents, and also the most common beverage brought to school, reported by $93.2 \%$. 
Table 3 Water and nutrition knowledge of the sample of elementaryschool children aged $8-14$ years ( $n$ 1049), London, Ontario, Canada, October-November 2016

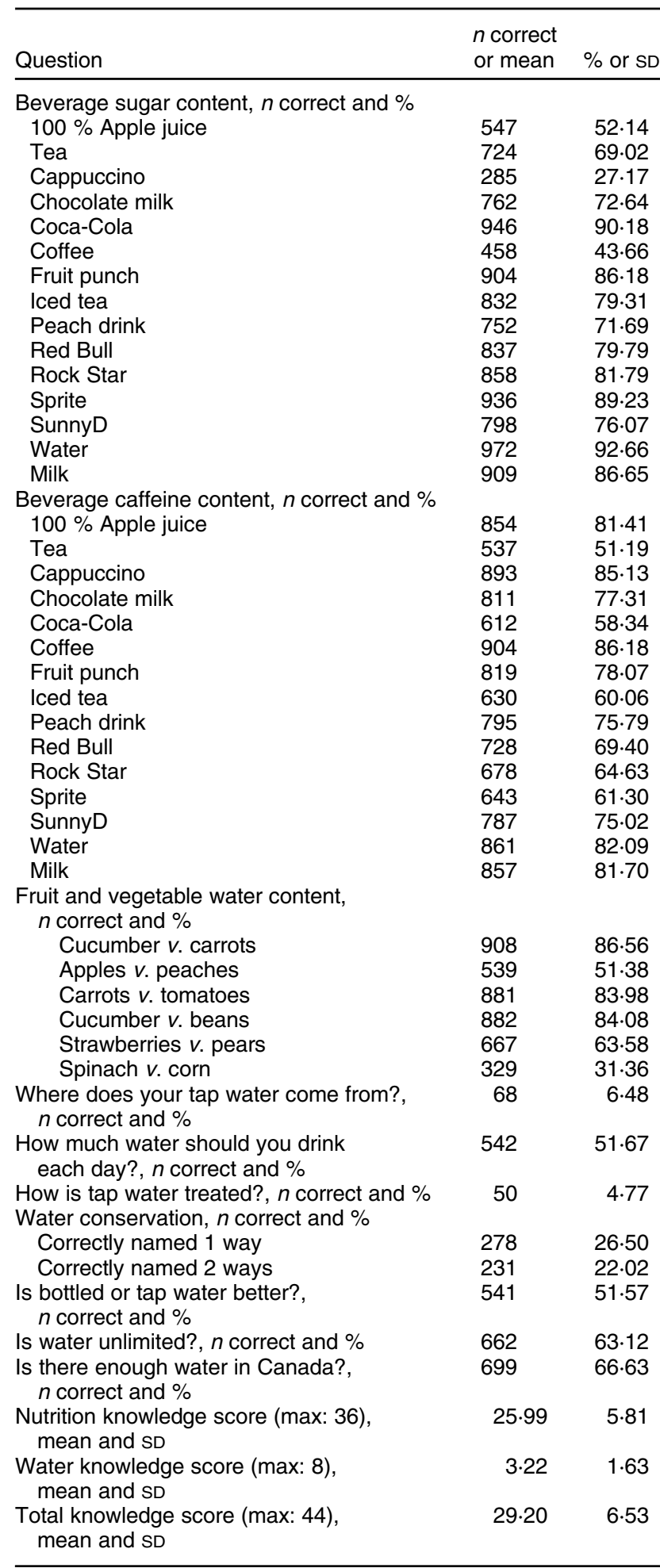

Just $14.5 \%$ of respondents participated in their school's milk programme. Fewer than one in five $(18.3 \%)$ were allowed to leave the school grounds at lunchtime.

\section{Water and nutrition knowledge}

The mean total knowledge score was $29 \cdot 2$ (SD 6.5) out of a possible 44 points (approximately $66.4 \%$ ), with mean subscale scores of 26.0 (SD 5.8) out of 36 for nutrition knowledge (approximately $72.2 \%$ ) and 3.2 (SD 1.6) out of 8 for water knowledge (approximately $40 \%$ ). Table 3 presents the proportion of students who responded correctly to each question. Children generally scored well on the questions related to nutritional aspects of different foods and beverages; however, knowledge of water, including its health benefits, conservation and treatment, was lacking. Just $6 \cdot 5 \%$ of respondents knew the origin of their tap water, and only $22 \%$ could name two ways to conserve water. Furthermore, just over half $(51.7 \%)$ of children knew how many cups of water they should consume in a day, and almost half ( $48.4 \%$ ) believed bottled water to be superior to tap water.

\section{Relationship between water and nutrition knowledge and beverage consumption habits}

The relationship between children's beverage consumption and water and nutrition knowledge is presented in Table 4. Higher total knowledge scores, along with higher water and nutrition subscale scores, were associated with significantly higher water and lower SSB consumption in both crude and adjusted analyses.

A one-point increase in total knowledge score was associated with a $0.34 \%$ (95\% CI $0.16,0.52 \% ; P=0.0002)$ increase in total daily beverage consumption attributable to water and a $0.33 \%(95 \%$ CI $-0.49,-0.18 \% ; P<0.0001)$ decrease in total daily beverage consumption attributable to SSB, adjusting for sociodemographic, dietary and behavioural factors. In looking at water and nutrition knowledge subscales separately, a one-point increase in water knowledge was associated with a $1 \cdot 12 \%$ (95\% CI 0.39, 1.85\%; $P=0.0026)$ increase in total daily beverage consumption attributable to water and a $1.41 \%$ (95\% CI $-2 \cdot 03,-0.79 \%$; $P<0.0001)$ decrease in total daily beverage consumption attributable to SSB, while a one-point increase in nutrition knowledge was associated with a $0.32 \%$ (95\% CI $0 \cdot 12$, $0.52 \% ; \quad P=0.0015)$ increase in total daily beverage consumption attributable to water and a $0.29 \%$ (95\% CI $-0.46,-0.12 \% ; P=0.0008)$ decrease in total daily beverage consumption attributable to SSB, adjusting for sociodemographic, dietary and behavioural factors.

\section{Determinants of water and sugar-sweetened beverage consumption and knowledge}

Tables 5-7 present the associations between water and SSB consumption and knowledge and various sociodemographic, dietary and behavioural factors. Older age, higher household income level and more educated parents/guardians were associated with higher total knowledge scores. 
Table 4 Relationship between knowledge score and proportion of water and sugar-sweetened beverage (SSB) consumption among the sample of elementary-school children aged 8-14 years ( $n$ 1049), London, Ontario, Canada, October-November 2016

\begin{tabular}{|c|c|c|c|c|c|c|}
\hline & \multicolumn{3}{|c|}{$\begin{array}{l}\text { Percentage of total beverage consumption } \\
\text { frequency attributable to water }\end{array}$} & \multicolumn{3}{|c|}{$\begin{array}{l}\text { Percentage of total beverage consumption } \\
\text { frequency attributable to SSB } \dagger\end{array}$} \\
\hline & $\beta$ & $95 \% \mathrm{Cl}$ & $P$ & $\beta$ & $95 \% \mathrm{Cl}$ & $P$ \\
\hline \multicolumn{7}{|c|}{ Total knowledge score } \\
\hline Unadjusted & 0.56 & $0.39,0.74$ & $<0.0001^{* * *}$ & -0.56 & $-0.72,-0.40$ & $<0.0001^{\star \star *}$ \\
\hline Model 1‡ & 0.51 & $0.32,0.70$ & $<0.0001^{\star * *}$ & -0.50 & $-0.67,-0.34$ & $<0.0001^{\star * \star}$ \\
\hline Model $2 \S$ & 0.37 & $0.19,0.56$ & $<0.0001^{\star \star *}$ & -0.37 & $-0.52,-0.21$ & $<0.0001^{\star \star \star}$ \\
\hline Model 3\| & 0.34 & $0.16,0.52$ & $0.0002^{\star * *}$ & -0.33 & $-0.49,-0.18$ & $<0.0001^{* * *}$ \\
\hline \multicolumn{7}{|c|}{ Water knowledge score } \\
\hline Unadjusted & 1.90 & $1 \cdot 17,2 \cdot 62$ & $<0.0001^{\star \star \star}$ & $-2 \cdot 12$ & $-2 \cdot 75,-1.49$ & $<0.0001^{\star \star \star}$ \\
\hline Model $1 \ddagger$ & 1.64 & $0.87,2 \cdot 41$ & $<0.0001^{\star * *}$ & $-1 \cdot 88$ & $-2 \cdot 55,-1 \cdot 22$ & $<0.0001^{* * *}$ \\
\hline Model $2 \S$ & $1 \cdot 11$ & $0.37,1.85$ & $0.0031^{\star *}$ & -1.36 & $-1.99,-0.74$ & $<0.0001^{\text {***}}$ \\
\hline Model 3\| & $1 \cdot 12$ & $0.39,1.85$ & $0.0026^{\star *}$ & -1.41 & $-2.03,-0.79$ & $<0.0001^{* * *}$ \\
\hline \multicolumn{7}{|c|}{ Nutrition knowledge score } \\
\hline Unadjusted & 0.56 & $0.35,0.76$ & $<0.0001^{\star \star \star}$ & -0.53 & $-0.71,-0.36$ & $<0.0001^{\star \star \star}$ \\
\hline Model 1‡ & 0.49 & $0.28,0.70$ & $<0.0001^{\star * *}$ & -0.46 & $-0.64,-0.28$ & $<0.0001^{* * *}$ \\
\hline Model $2 \S$ & 0.36 & $0.16,0.56$ & $0.0004^{\star \star \star}$ & -0.33 & $-0.51,-0.16$ & $0.0001^{* \star \star}$ \\
\hline Model 3\| & 0.32 & $0.12,0.52$ & $0.0015^{\star \star}$ & -0.29 & $-0.46,-0.12$ & $0.0008^{\star \star \star}$ \\
\hline
\end{tabular}

${ }^{\star \star} P<0.01,{ }^{* \star \star} P<0.001$

†SSB include fruit-flavoured drinks, regular pop, energy drinks and chocolate milk.

$\ddagger$ Model 1 adjusted for the sociodemographic characteristics sex, age, ethnicity, household income level, maximum household education, child living arrangement and parental work status.

$\S$ Model 2 added daily servings of fruits and vegetables and weekly junk food consumption frequency.

॥Model 3 added participation in school milk programme, permission to leave school grounds at lunchtime and frequency of refillable water bottle use.

Table 5 Predictors of water consumption among the sample of elementary-school children aged 8-14 years ( $n$ 1049), London, Ontario, Canada, October-November 2016

\begin{tabular}{|c|c|c|c|c|c|c|}
\hline & \multicolumn{3}{|c|}{ Crude } & \multicolumn{3}{|c|}{ Adjusted } \\
\hline & $\beta$ & $95 \% \mathrm{Cl}$ & $P$ & $\beta$ & $95 \% \mathrm{Cl}$ & $P$ \\
\hline \multicolumn{7}{|l|}{ Sociodemographic factors } \\
\hline Age & 0.66 & $-0.20,1.52$ & 0.1310 & 0.32 & $-0.49,1.13$ & 0.4494 \\
\hline Sex (ref. = female) & 5.42 & $3.03,7.82$ & $<0.0001^{\star \star *}$ & -3.68 & $-5.93,-1.43$ & $0.0016^{\star *}$ \\
\hline Ethnicity (ref. = Caucasian) & 0.99 & $-1.47,3.45$ & 0.4310 & 1.09 & $-1.45,3.62$ & 0.4414 \\
\hline Household income (ref. = high) & & & $0.0398^{*}$ & & & 0.5686 \\
\hline Low & $-5 \cdot 10$ & $-9.75,-0.44$ & & $-2 \cdot 81$ & $-8.06,2.43$ & \\
\hline Low-middle & $-3 \cdot 16$ & $-7.22,0.91$ & & -0.55 & $-4.65,3.56$ & \\
\hline High-middle & $-5 \cdot 57$ & $-9 \cdot 37,-1 \cdot 78$ & & $-2 \cdot 61$ & $-6 \cdot 22,1.01$ & \\
\hline Missing & $-1 \cdot 73$ & $-4 \cdot 64,1 \cdot 19$ & & -1.05 & $-3.90,1.81$ & \\
\hline Parental education (ref. $=$ college/university) & & & $0.0003^{\star \star \star}$ & & & $0.0160^{*}$ \\
\hline High school or less & $-5 \cdot 27$ & $-9.00,-1.55$ & & -2.93 & $-6.52,0.65$ & \\
\hline Graduate school & 3.20 & $-0.003,6.41$ & & 1.85 & $-1 \cdot 17,4 \cdot 87$ & \\
\hline Mother's employment status (ref. = employed) & -0.48 & $-3 \cdot 26,2 \cdot 29$ & 0.7323 & 0.96 & $-1.97,3.88$ & 0.6351 \\
\hline Father's employment status (ref. = employed) & 2.04 & $-3.05,7.13$ & 0.4322 & $2 \cdot 81$ & $-2 \cdot 27,7.89$ & 0.3307 \\
\hline Living arrangement (ref. = two-parent household) & & & $0.0002^{\star \star *}$ & & & $0.0074^{\star *}$ \\
\hline Single-parent household & $-5 \cdot 88$ & $-8.81,-2.95$ & & -3.49 & $-6 \cdot 36,-0.62$ & \\
\hline Other & $-6 \cdot 51$ & $-25.65,12.63$ & & -3.74 & $-21.41,13.94$ & \\
\hline \multicolumn{7}{|l|}{ Dietary factors } \\
\hline Daily servings of fruits and vegetables & -0.33 & $-0.94,0.27$ & 0.2800 & -0.71 & $-1.27,-0.15$ & $0.0115^{\star}$ \\
\hline Weekly junk food consumption frequency $\dagger$ & -0.71 & $-0.82,-0.60$ & $<0.0001^{\star \star *}$ & -0.65 & $-0.77,-0.54$ & $<0.0001^{\star * *}$ \\
\hline \multicolumn{7}{|l|}{ Behavioural factors } \\
\hline Participation in school milk programme (ref. $=$ no) & -4.51 & $-7 \cdot 88,-1 \cdot 13$ & $0.0088^{\star \star}$ & -4.03 & $-7 \cdot 15,-0.92$ & $0.0087^{* *}$ \\
\hline Permission to leave school grounds at lunchtime (ref. $=$ no) & -3.48 & $-6.57,-0.39$ & $0.0271^{*}$ & -2.93 & $-5.58,0.24$ & 0.0609 \\
\hline Frequency of water bottle use (ref. = always) & & & $0.0008^{* * *}$ & & & $0.0018^{\star *}$ \\
\hline Never & -4.52 & $-9.98,0.93$ & & $-4 \cdot 61$ & $-9.71,0.48$ & \\
\hline Rarely & $-6 \cdot 60$ & $-11.59,-1.61$ & & -5.59 & $-10 \cdot 30,-0.89$ & \\
\hline Sometimes & $-4 \cdot 15$ & $-7.39,-0.91$ & & $-3 \cdot 25$ & $-6 \cdot 29,-0 \cdot 21$ & \\
\hline Usually & 0.60 & $-2 \cdot 36,3.56$ & & 0.26 & $-2.48,3.00$ & \\
\hline
\end{tabular}

Ref, reference category.

${ }^{*} P<0.05,{ }^{* *} P<0.01,{ }^{* \star *} P<0.001$.

†Junk food includes sweetened cereal, cake/pie/doughnuts, potato chips, chocolate bars, pizza, French fries, hot dogs, ice cream, candy, granola bars and cookies. 
Table 6 Predictors of sugar-sweetened beverage (SSB) consumption among the sample of elementary-school children aged 8-14 years ( $n$ 1049), London, Ontario, Canada, October-November 2016

\begin{tabular}{|c|c|c|c|c|c|c|}
\hline & \multicolumn{3}{|c|}{ Crude } & \multicolumn{3}{|c|}{ Adjusted } \\
\hline & $\beta$ & $95 \% \mathrm{Cl}$ & $P$ & $\beta$ & $95 \% \mathrm{Cl}$ & $P$ \\
\hline \multicolumn{7}{|l|}{ Sociodemographic factors } \\
\hline Age & -0.89 & $-1.64,-0.15$ & $0.0189^{*}$ & -0.70 & $-1.39,-0.01$ & 0.0644 \\
\hline Sex (ref. $=$ female) & $-5 \cdot 65$ & $-7 \cdot 73,-3 \cdot 58$ & $<0.0001^{\star \star *}$ & 3.72 & $1 \cdot 79,5 \cdot 65$ & $<0.0001^{\star \star \star}$ \\
\hline Ethnicity (ref. = Caucasian) & -0.80 & $-2.95,1.35$ & 0.4652 & -0.48 & $-2 \cdot 66,1 \cdot 70$ & 0.5866 \\
\hline Household income (ref. = high) & & & 0.2759 & & & 0.2661 \\
\hline Low & $2 \cdot 25$ & $-1 \cdot 81,6 \cdot 30$ & & -1.72 & $-6 \cdot 22,2 \cdot 79$ & \\
\hline Low-middle & 2.45 & $-1 \cdot 10,6 \cdot 01$ & & -0.93 & $-4.46,2.59$ & \\
\hline High-middle & 4.95 & $1.65,8.26$ & & $2 \cdot 17$ & $-1.52,3.40$ & \\
\hline Missing & 1.76 & $-0.78,4.31$ & & 0.94 & $-1.52,3.40$ & \\
\hline Parental education (ref. $=$ college/university) & & & $0.0003^{\star \star *}$ & & & $0.0221^{*}$ \\
\hline High school or less & 4.64 & $1.36,7.92$ & & $2 \cdot 19$ & $-0.89,5.27$ & \\
\hline Graduate school & $-2 \cdot 82$ & $-5.61,-0.03$ & & -1.86 & $-4.43,0.71$ & \\
\hline Mother's employment status (ref. = employed) & 0.60 & $-1.88,3.08$ & 0.6349 & -0.61 & $-3.19,1.97$ & 0.5454 \\
\hline Father's employment status (ref. = employed) & $1 \cdot 13$ & $-3 \cdot 24,5 \cdot 51$ & 0.6113 & 1.39 & $-2.99,5.77$ & 0.6067 \\
\hline Living arrangement (ref. = two-parent household) & & & $0.0003^{\star \star \star}$ & & & $0.0119^{*}$ \\
\hline Single-parent household & 4.67 & $2 \cdot 13,7 \cdot 22$ & & 2.65 & $0 \cdot 19,5 \cdot 11$ & \\
\hline Other & -2.65 & $-19 \cdot 25,13.96$ & & -4.61 & $-19 \cdot 68,10.47$ & \\
\hline \multicolumn{7}{|l|}{ Dietary factors } \\
\hline Daily servings of fruits and vegetables & -0.60 & $-1 \cdot 14,-0.07$ & $0.0263^{*}$ & -0.24 & $-0.73,0.24$ & 0.3316 \\
\hline Weekly junk food consumption frequency $†$ & 0.66 & $0.56,0.76$ & $<0.0001^{\star \star *}$ & 0.61 & $-0.52,0.71$ & $<0.0001^{\star * \star}$ \\
\hline \multicolumn{7}{|l|}{ Behavioural factors } \\
\hline Participation in school milk programme (ref. $=$ no) & 4.41 & $1.48,7.35$ & $0.0032^{\star \star}$ & 3.75 & $1.07,6.43$ & $0.0065^{\star *}$ \\
\hline Permission to leave school grounds at lunchtime (ref. $=$ no) & $5 \cdot 18$ & $2.49,7.86$ & $0.0002^{\star \star \star}$ & $4 \cdot 73$ & $2 \cdot 23,7.22$ & $0.0002^{\star \star \star}$ \\
\hline Frequency of water bottle use (ref. = always) & & & $0.0004^{* * *}$ & & & $0.0096^{\star *}$ \\
\hline Never & $6 \cdot 11$ & $1.35,10 \cdot 87$ & & $5 \cdot 07$ & $0.73,9.41$ & \\
\hline Rarely & 4.89 & $0.49,9.28$ & & $2 \cdot 85$ & $-1 \cdot 20,6 \cdot 90$ & \\
\hline Sometimes & 3.46 & $0.64,6.29$ & & 1.59 & $-1.01,4 \cdot 19$ & \\
\hline Usually & 0.61 & $-1.97,3.18$ & & 0.26 & $-2 \cdot 07,2.59$ & \\
\hline
\end{tabular}

Ref, reference category.

${ }^{\star} P<0.05,{ }^{\star *} P<0.01,{ }^{\star \star *} P<0.001$.

†Junk food includes sweetened cereal, cake/pie/doughnuts, potato chips, chocolate bars, pizza, French fries, hot dogs, ice cream, candy, granola bars and cookies.

Table 7 Predictors of total knowledge score among the sample of elementary-school children aged 8-14 years ( $n$ 1049), London, Ontario, Canada, October-November 2016

\begin{tabular}{|c|c|c|c|c|c|c|}
\hline & \multicolumn{3}{|c|}{ Crude } & \multicolumn{3}{|c|}{ Adjusted } \\
\hline & $\beta$ & $95 \% \mathrm{Cl}$ & $P$ & $\beta$ & $95 \% \mathrm{Cl}$ & $P$ \\
\hline \multicolumn{7}{|l|}{ Sociodemographic factors } \\
\hline Age & 1.53 & $1.26,1.80$ & $<0.0001^{\star \star \star}$ & 1.51 & $1.25,1.78$ & $<0.0001^{\star \star \star}$ \\
\hline Sex (ref. = female) & -0.36 & $-1 \cdot 16,0.44$ & 0.3785 & -0.34 & $-1.09,0.41$ & 0.3784 \\
\hline Ethnicity (ref. = Caucasian) & -0.57 & $-1.39,0.24$ & 0.1687 & -0.01 & $-0.87,0.84$ & 0.7068 \\
\hline Household income (ref. = high) & & & $<0.0001^{\star \star *}$ & & & $0.0069^{\star *}$ \\
\hline Low & $-2 \cdot 88$ & $-4.41,-1.35$ & & -1.73 & $-3.52,0.05$ & \\
\hline Low-middle & $-2 \cdot 48$ & $-3 \cdot 82,-1 \cdot 14$ & & -1.65 & $-3.03,-0.28$ & \\
\hline High-middle & -0.80 & $-2.04,0.45$ & & -0.54 & $-0.54,0.63$ & \\
\hline Missing & -0.32 & $-1.28,0.64$ & & -0.02 & $-0.02,0.49$ & \\
\hline Parental education (ref. $=$ college/university) & & & $<0.0001^{* * *}$ & & & $<0.0001^{\star \star \star}$ \\
\hline High school or less & $-2 \cdot 74$ & $-3.98,-1.50$ & & $-2 \cdot 41$ & $-3 \cdot 64,-1 \cdot 19$ & \\
\hline Graduate school & 0.82 & $-0.24,1.88$ & & 0.58 & $-0.44,1.60$ & \\
\hline Mother's employment status (ref. = employed) & $-1 \cdot 10$ & $-2 \cdot 04,-0.17$ & $0.0211^{*}$ & -0.24 & $-1.25,0.77$ & 0.3262 \\
\hline Father's employment status (ref. = employed) & -0.88 & $-2 \cdot 61,0.85$ & 0.3195 & 0.17 & $-1.62,1.95$ & 0.9993 \\
\hline Living arrangement (ref. $=$ two-parent household) & & & $0.0379^{*}$ & & & 0.2256 \\
\hline Single-parent household & -0.99 & $-1.96,-0.01$ & & -0.38 & $-1.34,0.58$ & \\
\hline Other & $2 \cdot 02$ & $-4 \cdot 38,8 \cdot 42$ & & $3 \cdot 14$ & $-2 \cdot 83,9 \cdot 12$ & \\
\hline
\end{tabular}

Ref., reference category.

${ }^{\star} P<0.05,{ }^{\star \star} P<0.01,{ }^{\star \star \star} P<0.001$.

Higher water consumption was associated with being female, having a more educated parent/guardian, living in a two-parent/guardian household, consuming junk food less frequently, consuming fewer servings of fruits and vegetables, not participating in a school milk programme and using a refillable water bottle more frequently. 
Table 8 Sensitivity analysis, using non-imputed data (complete case analysis), for the relationship between knowledge score and proportion of water and sugar-sweetened beverage (SSB) consumption among the sample of elementary-school children aged 8-14 years ( $n$ 1049), London, Ontario, Canada, October-November 2016

\begin{tabular}{|c|c|c|c|c|c|c|}
\hline & \multicolumn{3}{|c|}{$\begin{array}{l}\text { Percentage of total beverage consumption } \\
\text { frequency attributable to water }\end{array}$} & \multicolumn{3}{|c|}{$\begin{array}{l}\text { Percentage of total beverage consumption } \\
\text { frequency attributable to SSB } \dagger\end{array}$} \\
\hline & $\beta$ & $95 \% \mathrm{Cl}$ & $P$ & $\beta$ & $95 \% \mathrm{Cl}$ & $P$ \\
\hline \multicolumn{7}{|c|}{ Total knowledge score } \\
\hline Unadjusted & 0.55 & $0.36,0.74$ & $<0.0001^{\star \star \star}$ & -0.56 & $-0.76,-0.37$ & $<0.0001^{\star \star \star}$ \\
\hline Model 1‡ & 0.49 & $0.24,0.74$ & $0.0001^{\star \star *}$ & -0.50 & $-0.69,-0.31$ & $<0.0001^{\star \star *}$ \\
\hline Model $2 \S$ & 0.36 & $0.13,0.60$ & $0.0026^{\star \star}$ & -0.38 & $-0.53,-0.23$ & $<0.0001^{\star \star *}$ \\
\hline Model 3|| & 0.34 & $0.06,0.61$ & $0.0160^{*}$ & -0.33 & $-0.51,-0.15$ & $0.0003^{\star \star \star}$ \\
\hline \multicolumn{7}{|c|}{ Water knowledge score } \\
\hline Unadjusted & 1.87 & $0.97,2.78$ & $<0.0001^{\star \star \star}$ & $-2 \cdot 13$ & $-2 \cdot 67,-1 \cdot 59$ & $<0.0001^{\star \star *}$ \\
\hline Model 1‡ & 1.91 & $0.76,0.43$ & $0 \cdot 0116^{*}$ & -1.99 & $-2 \cdot 83,-1 \cdot 15$ & $<0.0001^{\star \star \star}$ \\
\hline Model $2 \S$ & 1.34 & $0.06,2.63$ & $0.0407^{*}$ & -1.34 & $-2 \cdot 01,-0.67$ & $<0.0001^{\star * *}$ \\
\hline Model 3|| & 1.31 & $-0.50,2.67$ & 0.0599 & -1.22 & $-1.91,-0.54$ & $0.0005^{\star \star *}$ \\
\hline \multicolumn{7}{|c|}{ Nutrition knowledge score } \\
\hline Unadjusted & 0.55 & $0.32,0.77$ & $<0.0001^{\star \star *}$ & -0.54 & $-0.76,-0.31$ & $<0.0001^{\star \star *}$ \\
\hline Model 1‡ & 0.44 & $0.18,0.70$ & $0.0009^{* \star *}$ & -0.44 & $-0.64,-0.24$ & $<0.0001^{\star \star \star}$ \\
\hline Model $2 \S$ & 0.33 & $0.07,0.58$ & $0.0112^{*}$ & -0.36 & $-0.52,-0.20$ & $<0.0001^{\star * *}$ \\
\hline Model 3\| & 0.30 & $0.01,0.59$ & $0.0446^{*}$ & -0.30 & $-0.48,-0.12$ & $0.0010^{* * *}$ \\
\hline
\end{tabular}

${ }^{*} P<0.05,{ }^{* *} P<0.01,{ }^{* * *} P<0.001$.

†SSB include fruit-flavoured drinks, regular pop, energy drinks and chocolate milk.

¥Model 1 adjusted for the sociodemographic characteristics sex, age, ethnicity, household income level, maximum household education level, child living arrangement and parental employment status.

$\S$ Model 2 added daily servings of fruits and vegetables and weekly junk food consumption frequency.

॥Model 3 added participation in a school milk programme, permission to leave school grounds at lunchtime and frequency of refillable water bottle use.

Higher SSB consumption was associated with being male, having a less educated parent/guardian, consuming junk food more frequently, living in a single-parent/guardian household, participating in a school milk programme, using a refillable water bottle less frequently and being allowed to leave the school grounds at lunchtime.

\section{Sensitivity analyses}

A sensitivity analysis was performed using non-imputed data only. The results of this complete case analysis are presented in Table 8. Although effect estimates were less precise, they were similar in size and direction, and all associations remained significant, except for that between water knowledge score and percentage of total daily beverage consumption attributable to water $(\beta=0.30 ; 95 \%$ $\mathrm{CI}-0.50,2 \cdot 67 ; P=0.0599)$. An additional sensitivity analysis using absolute beverage consumption frequencies rather than proportions was also conducted and is presented in Table 9. Again, trends were similar, with a positive association observed between knowledge and water consumption in adjusted models and a negative association observed between knowledge and SSB consumption; however, only the estimates for SSB consumption were statistically significant.

\section{Discussion}

The present study described the beverage consumption habits of a sample of elementary-school children in London, Ontario, Canada, and examined the association between knowledge and water and SSB intake. As far as we know, the present study is the first to specifically evaluate the relationship between knowledge and beverage consumption.

\section{Knowledge is a determinant of beverage consumption habits}

Our results indicate that knowledge is a significant predictor of beverage consumption habits, with higher-scoring children consuming a higher proportion of water and a lower proportion of SSB than their lower-scoring peers. This association remained significant when nutrition and water knowledge sub-scores were analysed separately. These findings are consistent with the previous research investigating the association between knowledge and dietary intake in this population, which identified a weak positive correlation overall. A survey performed in Iceland among 11-year-old children, for example, found that knowledge was a significant determinant of fruit and vegetable consumption $^{(41)}$, while a Japanese study identified a strong association between nutrition knowledge and vegetable intake in elementary-school children ${ }^{(39)}$. A positive correlation between nutrition knowledge and eating behaviour was additionally observed in American children, particularly as they increased in age ${ }^{(42)}$, and a study of Sicilian children found that nutrition knowledge was negatively associated with a number of unhealthy foods including sugary drinks ${ }^{(40)}$.

These findings are also supported by a qualitative study examining the perceptions and determinants of SSB consumption in London elementary-school children, in which participants identified improving knowledge as a necessary 
Table 9 Sensitivity analysis, using absolute frequencies of beverage consumption, for the relationship between knowledge score and proportion of water and sugar-sweetened beverage (SSB) consumption among the sample of elementary-school children aged 8-14 years ( $n$ 1049), London, Ontario, Canada, October-November 2016

\begin{tabular}{|c|c|c|c|c|c|c|}
\hline & \multicolumn{3}{|c|}{$\begin{array}{l}\text { Percentage of total beverage consumption } \\
\text { frequency attributable to water }\end{array}$} & \multicolumn{3}{|c|}{$\begin{array}{l}\text { Percentage of total beverage consumption } \\
\text { frequency attributable to SSB } \dagger\end{array}$} \\
\hline & $\beta$ & $95 \% \mathrm{Cl}$ & $P$ & $\beta$ & $95 \% \mathrm{Cl}$ & $P$ \\
\hline \multicolumn{7}{|c|}{ Total knowledge score } \\
\hline Unadjusted & -0.0002 & $-0.02,0.02$ & 0.9840 & -0.15 & $-0.19,-0.11$ & $<0.0001^{\star \star \star}$ \\
\hline Model $1 \ddagger$ & 0.002 & $-0.02,0.02$ & 0.8024 & -0.13 & $-0.18,-0.09$ & $<0.0001^{\text {*** }}$ \\
\hline Model $2 \S$ & 0.007 & $-0.01,0.03$ & 0.5059 & -0.08 & $-0.12,-0.05$ & $<0.0001^{\star \star \star *}$ \\
\hline Model 3\| & 0.006 & $-0.01,0.02$ & 0.5551 & -0.08 & $-0.11,-0.04$ & $<0.0001^{\star \star \star}$ \\
\hline \multicolumn{7}{|c|}{ Water knowledge score } \\
\hline Unadjusted & 0.04 & $-0.03,0.11$ & 0.3097 & -0.42 & $-0.58,-0.27$ & $<0.0001^{\star \star \star}$ \\
\hline Model $1 \ddagger$ & 0.04 & $-0.03,0.12$ & 0.2816 & -0.35 & $-0.52,-0.18$ & $<0.0001^{\text {*** }}$ \\
\hline Model $2 \S$ & 0.05 & $-0.02,0.13$ & 0.1656 & $-0 \cdot 16$ & $-0.30,-0.02$ & $0.0260^{\star}$ \\
\hline Model 3\| & 0.05 & $-0.02,0.13$ & $0 \cdot 1820$ & -0.17 & $-0.30,-0.03$ & $0.0194^{\star}$ \\
\hline \multicolumn{7}{|c|}{ Nutrition knowledge score } \\
\hline Unadjusted & -0.003 & $-0.02,0.02$ & 0.7980 & -0.15 & $-0.20,-0.11$ & $<0.0001^{* * *}$ \\
\hline Model $1 \ddagger$ & 0.0002 & $-0.02,0.02$ & 0.9863 & -0.13 & $-0.18,-0.09$ & $<0.0001^{\text {*** }}$ \\
\hline Model $2 \S$ & 0.004 & $-0.02,0.02$ & 0.7109 & -0.09 & $-0.13,-0.05$ & $<0.0001^{\star \star \star}$ \\
\hline Model 3\| & 0.003 & $-0.02,0.02$ & 0.7744 & -0.08 & $-0.12,-0.04$ & $<0.0001^{* * *}$ \\
\hline
\end{tabular}

${ }^{\star} P<0.05,{ }^{* \star *} P<0.001$

†SSB include fruit-flavoured drinks, regular pop, energy drinks and chocolate milk.

†Model 1 adjusted for the sociodemographic characteristics sex, age, ethnicity, household income level, maximum household education level, child living arrangement and parental employment status.

$\S$ Model 2 added daily servings of fruits and vegetables and weekly junk food consumption frequency.

$\|$ Model 3 added participation in a school milk programme, permission to leave school grounds at lunchtime and frequency of refillable water bottle use.

strategy to help reduce their intake of sugary drinks ${ }^{(53)}$. Children specifically noted the need for hands-on and engaging educational programmes, and believed that education should be incorporated into the curriculum as early as possible ${ }^{(53)}$.

\section{Water and nutrition knowledge is limited}

Our results also provide valuable insights into the gaps in knowledge that exist within this population, which can be used to develop more effective interventions. For example, although we observed a significant association between knowledge and practice, knowledge in our sample was relatively low overall, with children scoring an average of $66 \%$ in total and $72 \%$ and $40 \%$ on the nutrition and water subscales, respectively. Indeed, an evaluation of the survey results demonstrated that children had very little knowledge of the water treatment system or water conservation, and almost half were unaware of the amount of water they should consume in a day.

Even more concerning, a lack of knowledge about the safety of tap water was identified and the belief that bottled water was superior to tap was widespread. Although London's tap water is of high quality and is rigorously monitored ${ }^{(54)}$, these findings are in line with the existing literature, which has identified negative perceptions of tap water among North American children and adolescents pertaining to taste and cleanliness ${ }^{(55,50)}$, and may explain the low number of children reporting consuming tap water at home in our study. Given that larger point estimates were observed for water knowledge than nutrition knowledge, suggesting that water knowledge may have a greater influence on drinking behaviours than nutrition knowledge, future education programmes to improve children's drinking habits must incorporate lessons on water specifically and should attempt to dispel negative attitudes and beliefs about water in order to achieve the best possible results.

\section{Children have poor dietary habits overall}

Corresponding with the low levels of knowledge observed, children in our sample consumed relatively low proportions of water, accounting for approximately $39.8 \%$ of their total daily beverage intake, and high proportions of SSB, accounting for approximately $22.0 \%$ of their total daily beverage intake. This substantially exceeds the guidelines set by the American Heart Association, which has recommended that children consume no more than 8 fluid ounces $(237 \mathrm{ml})$ of SSB per week ${ }^{(57)}$, approximately $1.7-$ $2.25 \%$ of the total daily fluid intake recommendations. These findings, however, are in agreement with other studies of SSB intake in Canadian children and adolescents, which have also observed an overconsumption of these beverages $^{(15,58,59)}$. A survey of youths from three Canadian cities, for instance, found that $80 \%$ of respondents consumed at least one SSB daily, and $44 \%$ consumed three or more ${ }^{(60)}$. This is comparable to our sample, of which $79.4 \%$ reported consuming an SSB at least once daily, and half $49.6 \%$ reported consuming an SSB three or more times daily.

In addition to knowledge, we identified several independent predictors of more frequent SSB consumption which can potentially be used to identify individuals who may benefit most from interventions. These included male sex, more frequent junk food consumption, living in a 
single-parent household, having less educated parents, participating in a school milk programme, using a refillable water bottle less frequently and having permission to leave school grounds at lunchtime. Independent predictors of more frequent water consumption included female sex, having more educated parents, living in a two-parent household, consuming junk food less frequently, having fewer daily servings of fruits and vegetables, not participating in a school milk programme and using a refillable water bottle more frequently. These factors are in line with those examined in previous research, except the observed association between more frequent water consumption and fewer servings of fruits and vegetables. While this seems counterintuitive, with water and fruit and vegetable intake both markers of a healthy diet, children who consume more fruits and vegetables may get more of their daily water requirements through foods, which may translate to drinking less overall to compensate. We were also surprised to find that milk programme participation was negatively associated with water consumption but positively with SSB consumption. Although the effect of milk provision on water and SSB intake has not been studied directly in previous literature, we were expecting that children who participated in their school's milk programme would consume less of both beverages due to displacement caused by increased milk consumption. Interestingly, this was the case for water but not SSB. This could indicate that most children who participated in the programme receive chocolate milk rather than white, which was considered an SSB in the present study and thus potentially drove the observed association.

Along with poor beverage consumption habits, we also observed suboptimal diet quality in this sample. Just under $30 \%$ of children met Canada's Food Guide recommendation of six or more servings of fruits and vegetables daily, and junk foods such as candy, pizza and cake were consumed more than twice daily, on average. This is in line with previous studies of children's diets. For example, an examination of 2004 CCHS data indicated that $65 \%$ of 9-13-year-old children did not meet the recommended servings of fruits and vegetables $^{(61)}$, while another study found that just $26 \%$ of Canadians met the minimum daily fruit and vegetable servings for their age-sex group and that, in adolescents and teens, this number dropped to less than $20 \%{ }^{(62)}$.

\section{Implications for policy and practice}

Our results support the continued implementation of education programmes as a potentially effective strategy for reducing SSB consumption and/or increasing water consumption in child and adolescent populations. This is encouraging, as education interventions are cost-effective and easy to implement, compared with environmental and policy interventions, and are also highly reproducible, with successful programmes being adaptable for different populations and different settings. Given that children spend the majority of their waking hours in school, this is the ideal environment through which to deliver standardized evidence-based programmes targeting healthy behaviours ${ }^{(63)}$. Furthermore, when delivered in the school environment, education interventions are capable of reaching many children at once and may serve as an equalizer, minimizing differences in knowledge and access to information between children of different socio-economic statuses and backgrounds ${ }^{(64,65)}$. This is particularly important as we observed that, in addition to age, household income level and parental education were significant predictors of baseline knowledge in this population.

It must be noted, however, that although we identified a statistically significant association between high knowledge scores and healthier beverage consumption habits, the observed effects were small in magnitude and thus improvements in knowledge may not translate to clinically significant improvements in behaviour. Indeed, this may explain why education-only interventions to improve beverage consumption habits are often unsuccessful in the long term ${ }^{(26,28,66-73)}$. Further research is therefore needed to understand how to maximize the effect of education interventions in order to capitalize on the association between knowledge and behaviour.

\section{Limitations}

Our results should be interpreted in light of several limitations. First of all, although the study targeted children residing in disadvantaged neighbourhoods throughout the city of London, the descriptive statistics of the sample as presented in Table 1 reveal that only a small proportion ( $8.10 \%$ ) of respondents came from low-income households and just $11.89 \%$ of parents had not completed postsecondary education. This is not representative of the overall population of the neighbourhoods under study and likely reflects selection bias, wherein children from more disadvantaged households were less likely to enrol in the study, potentially due to language barriers or lower parental education, which may have limited a parent's ability to understand the consent form and parent survey.

Second, it is important to note that, in addition to knowledge, there are a number of other factors that influence children's food and beverage choices including taste preferences, advertising and parental control ${ }^{(53)}$. Given that the participants evaluated in the current study were relatively young, ranging from 8 to 14 years of age, parental control was likely a major determinant of dietary intake in those children who are restricted to what is available to them at home. Indeed, a number of studies have identified the importance of parental knowledge and maternal knowledge specifically on children's diet quality, finding that children with more knowledgeable parents had better diets ${ }^{(39,40)}$. Although our study was not able to assess parental knowledge, we did measure parental education, which can be considered a proxy. We found that higher parental education was associated with a significantly higher percentage of total daily beverage 
consumption attributable to water and with higher total knowledge scores for children; however, no significant association with SSB intake was observed.

Additionally, the ambiguous 'times per day' measure of water and SSB consumption, as opposed to a standardized volume measure such as cups or millilitres per day, may have been reported differently by each child, potentially resulting in measurement error. We attempted to correct for this by converting absolute frequencies into proportions in order to standardize responses. Moreover, some studies have suggested that children may have difficulties estimating volumes and portion sizes ${ }^{(74-77)}$, making instance frequencies the better choice for assessing beverage intake in this population. Indeed, instances have been used in the past in similar studies in child populations ${ }^{(27,33,40,78,79)}$.

Furthermore, as with most studies on children's dietary behaviours, our study used self-reported dietary data, which are vulnerable to recall bias and may be inaccurate, especially in children. Self-reported measures can be useful in that they are more suited to assessing usual intake, however, whereas observations and other objective measures assess recent intake, and there is also no risk of children changing their behaviours because they know they are being observed when using self-reported compared with objective measures $^{(80)}$.

Finally, the present study was cross-sectional. This prevents us from establishing temporality, and the direction of the relationship between knowledge and beverage consumption habits cannot be discerned.

\section{Conclusions}

In the present cross-sectional study of schoolchildren in Southwestern Ontario, we provided new evidence of an association between dietary intake and knowledge. We were able to demonstrate that children with higher knowledge scores had significantly healthier beverage consumption habits; however, knowledge in our sample was low overall, which was reflected in water and SSB intake. Future interventions to increase water and/or decrease SSB consumption in young children should therefore target water and nutrition knowledge through education programmes, as they may be effective at changing behaviour. Additional research is required to evaluate whether or not changes in knowledge actually yield clinically significant improvements in behaviour in practice, and should investigate the optimal characteristics of education interventions so that a framework can be developed for use in a variety of settings, populations and contexts.

\section{Acknowledgements:}

Acknowledgements: The authors gratefully thank the students and families who participated in this study, along with the teachers and principals of each school and the research boards from the Thames Valley District School Board and the London District Catholic School Board. They would also like to acknowledge the many research assistants and volunteers from the Human Environments Analysis Laboratory who helped with data collection and entry for the HKCC project, as well as the invaluable contributions of $\mathrm{Dr}$ Andrew Clark, who coordinated the project, and Dr Piotr Wilk, who assisted with the statistical techniques. Financial support: This study was jointly funded by the Ontario Ministry of Health and Long Term Care as part of the City of London's Healthy Kids Community Challenge (HKCC) and the Children's Health Foundation through the Children's Health Research Institute. Additionally, the Canadian Institute of Health Research (CIHR) provided graduate student funding in the form of a Canada Graduate Scholarship (CGS-M). Funders did not have any role in the study design, data collection/analysis, writing or publication of this study. Conflict of interest: None. Authorship: B.R.I. assisted with data collection and was responsible for the analysis of the data and the writing of the manuscript. M.R.S. provided input on the analysis and read and reviewed the manuscript. J.A.G. is Principal Investigator of the project and conceived of and designed the study, formulated the research question, provided input on the analysis, and read and reviewed the manuscript. Ethics of human subject participation: This study was conducted according to the guidelines laid down in the Declaration of Helsinki and all procedures involving human subjects were approved by Western University's NonMedical Human Research Ethics Board (NM108328) and the research boards of the Thames Valley District School Board and the London District Catholic School Board. Parents/guardians provided written informed consent to participate in the study and parental consent for their children to participate. All child participants provided written assent to complete the survey.

Author ORCID. (iD) Jason A Gilliland, 0000-00022909-2178.

\section{References}

1. Roberts KC, Shields M, de Groh M et al. (2012) Overweight and obesity in children and adolescents: results from the 2009 to 2011 Canadian Health Measures Survey. Health Rep 23, $37-41$.

2. Rao DP, Kropac E, Do MT et al. (2016) Childhood overweight and obesity trends in Canada. Health Promot Chronic Dis Prev Can 36, 194-199.

3. World Health Organization. (2012) Population Based Approaches to Childhood Obesity Prevention. Geneva: WHO.

4. Cawley J (2010) The economics of childhood obesity. Health Aff (Millwood) 29, 364-371.

5. Keller A \& Bucher Della Torre S (2015) Sugar-sweetened beverages and obesity among children and adolescents: a review of systematic literature reviews. Obes Facts 11, $338-346$ 
6. Trumbo PR \& Rivers CR (2014) Systematic review of the evidence for an association between sugar-sweetened beverage consumption and risk of obesity. Nutr Rev 72, 566-574.

7. Malik VS, Pan A, Willett WC et al. (2013) Sugar-sweetened beverages and weight gain in children and adults: a systematic review and meta-analysis. Am J Clin Nutr 94, 1084-1102.

8. Malik VS, Willett WC \& Hu FB (2009) Sugar-sweetened beverages and BMI in children and adolescents: reanalyses of a meta-analysis. Am J Clin Nutr 89, 438-439.

9. Gibson S (2008) Sugar-sweetened soft drinks and obesity: a systematic review of the evidence from observational studies and interventions. Nutr Res Rev 21, 134-147.

10. Ludwig DS, Peterson KE \& Gortmaker SL (2001) Relation between consumption of sugar-sweetened drinks and childhood obesity: a prospective, observational analysis. Lancet 357, 505-508.

11. Mullen M \& Shield JE (2017) Water: How Much Do Kids Need? http://www.eatright.org/resource/fitness/sports-andperformance/hydrate-right/water-go-with-the-flow (accessed March 2018).

12. Lee RM, Okechukwu C, Emmons KM et al. (2014). Impact of implementation factors on children's water consumption in the Out-of-School Nutrition and Physical Activity group-randomized trial. New Dir Youth Dev 143, 79-101.

13. Kant AK \& Graubard BI (2010) Contributors of water intake in US children and adolescents: associations with dietary and meal characteristics - National Health and Nutrition Examination Survey 2005-2006. Am J Clin Nutr 92, 887-896.

14. Drewnowski A, Rehm CD \& Constant F (2013) Water and beverage consumption among children age $4-13 y$ in the United States: analyses of 2005-2010 NHANES data. Nutr J 12, 85 .

15. Garriguet D (2008) Beverage consumption of children and teens. Health Rep 19, 17-22.

16. Hu FB (2013) Resolved: there is sufficient scientific evidence that decreasing sugar-sweetened beverage consumption will reduce the prevalence of obesity and obesity-related diseases. Obes Rev 14, 606-619.

17. Te Morenga L, Mallard S \& Mann J (2012) Dietary sugars and body weight: systematic review and meta-analyses of randomised controlled trials and cohort studies. BMJ 346, e7492.

18. Langlois K \& Garriguet D (2011) Sugar consumption among Canadians of all ages. Health Rep 22, 23-27.

19. Avery A, Bostock L \& McCullough F (2015) A systematic review investigating interventions that can help reduce consumption of sugar-sweetened beverages in children leading to changes in body fatness. J Hum Nutr Diet 28, Suppl. 1, 52-64.

20. Angelopoulos P, Tsitsas G, Milionis H et al. (2010) Effect of a school based intervention programme on anthropometric and clinical indices among primary schoolchildren: the children study. Diabetes Obes Metab 12, 67.

21. James J (2004) Preventing childhood obesity by reducing consumption of carbonated drinks: cluster randomised controlled trial. BMJ 328, 1237-1243.

22. Rausch Herscovici C, Kovalskys I \& De Gregorio MJ (2013) Gender differences and a school-based obesity prevention program in Argentina: a randomized trial. Rev Panam Salud Publica 34, 75-82.

23. Mantziki K, Renders CM, Vassilopoulos A et al. (2016) Inequalities in energy-balance related behaviours and family environmental determinants in European children: changes and sustainability within the EPHE evaluation study. Int $J$ Equity Health 15, 160.

24. Muckelbauer R, Gortmaker SL, Libuda L et al. (2016) Changes in water and sugar-containing beverage consumption and body weight outcomes in children. Br J Nutr 115, 2057-2066.

25. Porepa M, Chan M, Huber J et al. (2016) Creating a studentled health magazine with an urban, multicultural, resource- restricted elementary school: approach, process and impact. Paediatr Child Health 21, 119-122.

26. Kipping RR, Howe LD, Jago R et al. (2014) Effect of intervention aimed at increasing physical activity, reducing sedentary behaviour, and increasing fruit and vegetable consumption in children: Active for Life Year 5 (AFLY5) school based cluster randomised controlled trial. BMJ 348, g3256.

27. Korwanich K, Sheiham A, Srisuphan W et al. (2008) Promoting healthy eating in nursery school children: a quasiexperimental intervention study. Health Educ J 67, 16-30.

28. Rosário R, Araújo A, Oliveira B et al. (2013) Impact of an intervention through teachers to prevent consumption of low nutrition, energy-dense foods and beverages: a randomized trial. Prev Med 57, 20-25.

29. Sharma SV, Markham C, Chow J et al. (2016) Evaluating a school-based fruit and vegetable co-op in low-income children: a quasi-experimental study. Prev Med 91, 8-17.

30. Steyn NP, De Villiers A, Gwebushe N et al. (2015) Did HealthKick, a randomised controlled trial primary school nutrition intervention improve dietary quality of children in low-income settings in South Africa? BMC Public Health 15, 948 .

31. Taylor RW, McAuley KA, Barbezat W et al. (2007) APPLE Project: 2-y findings of a community-based obesity prevention program in primary school-age children. Am J Clin Nutr 86, 735-742.

32. van de Gaar VM, Jansen W, van Grieken A et al. (2014) Effects of an intervention aimed at reducing the intake of sugar-sweetened beverages in primary school children: a controlled trial. Int J Behav Nutr Phys Act 11, 98.

33. Cunha DB, Souza B, Pereira RA et al. (2013) Effectiveness of a randomized school-based intervention involving families and teachers to prevent excessive weight gain among adolescents in Brazil. PLoS One 8, e57498.

34. Maynard M, Gunnell D, Ness AR et al. (2006) What influences diet in early old age? Prospective and crosssectional analyses of the Boyd Orr cohort. Eur J Public Health 16, 316-324.

35. Mikkilä V, Räsänen L, Raitakari OT et al. (2005) Consistent dietary patterns identified from childhood to adulthood: the Cardiovascular Risk in Young Finns Study. Br J Nutr 93, 923-931.

36. World Health Organization (2003) Diet, Nutrition and the Prevention of Chronic Diseases. Report of a Joint WHO/ FAO Expert Consultation. WHO Technical Report Series no. 916. http://apps.who.int/iris/bitstream/handle/10665/ 42665/WHO_TRS_916.pdf;jsessionid=85265663024BE5EC0 48680E86D336167?sequence $=1$ (accessed March 2018).

37. Breinbauer C \& Maddaleno M (2005) Youth: Choices and Change: Promoting Healthy Behaviors in Adolescents. http://iris.paho.org/xmlui/handle/123456789/708 (accessed March 2019).

38. Spronk I, Kullen C, Burdon C et al. (2014) Relationship between nutrition knowledge and dietary intake. Br J Nutr 111, 1713-1726.

39. Asakura K, Todoriki H \& Sasaki S (2017) Relationship between nutrition knowledge and dietary intake among primary school children in Japan: combined effect of children's and their guardians' knowledge. J Epidemiol 10, 483-491.

40. Grosso G, Mistretta A, Turconi G et al. (2013) Nutrition knowledge and other determinants of food intake and lifestyle habits in children and young adolescents living in a rural area of Sicily, South Italy. Public Health Nutr 16, 1827-1836.

41. Kristjansdottir AG, Thorsdottir I, De Bourdeaudhuij I et al. (2006) Determinants of fruit and vegetable intake among 11 -year-old schoolchildren in a country of traditionally low fruit and vegetable consumption. Int J Behav Nutr Phys Act 3, 41 . 
42. Pirouznia M (2001) The association between nutrition knowledge and eating behavior in male and female adolescents in the US. Int I Food Sci Nutr 52, 127-132.

43. Pirouznia M (2000) The correlation between nutrition knowledge and eating behavior in an American school: the role of ethnicity. Nutr Health 14, 89-107.

44. Reinehr T, Kersting M, Chahda C et al. (2003) Nutritional knowledge of obese compared to non obese children. Nutr Res 23, 645-649.

45. Gilliland JA (2016) Healthy Kids Community Challenge City of London: Community Needs Assessment. https://issuu.com/ wrroberts/docs/london_cna_final_final (accessed May 2018).

46. Mitchell CA, Clark AF \& Gilliland JA (2016) Built environment influences of children's physical activity: examining differences by neighbourhood size and sex. Int J Environ Res Public Health 13, 130-144.

47. Dietitians of Canada (2016) Taxation and Sugar-Sweetened Beverages: Position of Dietitians of Canada. https://www. dietitians.ca/Downloads/Public/DC-Position-SSBs-and-taxation. aspx (accessed May 2018).

48. Centers for Disease Control and Prevention (2010) The CDC Guide to Strategies for Reducing the Consumption of SugarSweetened Beverages. https://stacks.cdc.gov/view/cdc/ 51532 (accessed March 2019).

49. Gill JMR \& Sattar N (2014) Fruit juice: just another sugary drink? Lancet Diabetes Endocrinol 2, 444-445.

50. Statistics Canada, Health Statistics Division (2002) Canadian Community Health Survey (CCHS) Cycle 1.2: Derived Variable (DV) Specifications. http://www23.statcan. gc.ca/imdb-bmdi/pub/document/5015_D8_T9_V1-eng.pdf (accessed January 2018).

51. Bodner TE (2011) What improves with increased missing data imputations? Struct Equ Modeling 15, 651-675.

52. White IR, Royston P \& Wood AM (2011) Multiple imputation using chained equations: issues and guidance for practice. Stat Med 30, 377-399.

53. Battram DS, Piché L, Beynon C et al. (2016) Sugar-sweetened beverages: children's perceptions, factors of influence, and suggestions for reducing intake. J Nutr Educ Behav 48, $27-34$

54. City of London (2018) Water Quality. https://www.london. $\mathrm{ca} /$ residents/Water/Water-System/Pages/Water-Quailty.aspx (accessed July 2018).

55. Onufrak SJ, Park S, Sharkey JR et al. (2014) Perceptions of tap water and school water fountains and association with intake of plain water and sugar-sweetened beverages. J Sch Health 84, 195-204

56. Huerta-Saenz L, Irigoyen M, Benavides J et al. (2012) Tap or bottled water: drinking preferences among urban minority children and adolescents. J Community Health 37, 54-58.

57. Johnson RK, Appel LJ, Brands M et al. (2009) Dietary sugars intake and cardiovascular health: a scientific statement from the American Heart Association. Circulation 120, 1011-1020.

58. Danyliw AD, Vatanparast H, Nikpartow N et al. (2011) Beverage intake patterns of Canadian children and adolescents. Public Health Nutr 14, 1961-1969.

59. Danyliw AD, Vatanparast H, Nikpartow N et al. (2012) Beverage patterns among Canadian children and relationship to overweight and obesity. Appl Physiol Nutr Metab 37, 900-906.

60. Vanderlee L, Manske S, Murnaghan D et al. (2014) Sugarsweetened beverage consumption among a subset of Canadian youth. J Sch Health 84, 168-176.

61. Garriguet D (2007) Canadians' eating habits. Health Rep 18, $17-32$.

62. Black JL \& Billette J-M (2013) Do Canadians meet Canada's Food Guide's recommendations for fruits and vegetables? Appl Physiol Nutr Metab 38, 234-242.
63. Flynn MAT, McNeil DA, Maloff B et al. (2006) Reducing obesity and related chronic disease risk in children and youth: a synthesis of evidence with 'best practice' recommendations. Obes Rev 7, 7-66.

64. Hahn RA \& Truman BI (2015) Education improves public health and promotes health equity. Int J Health Serv 45, 657-678.

65. Cohen AK \& Syme SL (2013) Education: a missed opportunity for public health intervention. Am J Public Health 103, 997-1001.

66. Al Khalifah RA, Mok E, Legault L et al. (2016) Using '5, 2, 1, 0' to promote healthy active living among school-age children attending a paediatric resident clinic: a prospective study. Paediatr Child Health 21, 43-47.

67. Anttonen V, Seppä L, Niinimaa A et al. (2011) Dietary and oral hygiene intervention in secondary school pupils. Int $J$ Paediatr Dent 21, 81-88.

68. Bayer O, von Kries R, Strauss A et al. (2009) Short- and midterm effects of a setting based prevention program to reduce obesity risk factors in children: a cluster-randomized trial. Clin Nutr 28, 122-128.

69. Burrows T, Janet WM \& Collins CE (2011) Long-term changes in food consumption trends in overweight children in the HIKCUPS intervention. J Pediatr Gastroenterol Nutr 53, 543-547.

70. Ezendam NPM, Brug J \& Oenema A (2012) Evaluation of the web-based computer-tailored FATaintPHAT intervention to promote energy balance among adolescents: results from a school cluster randomized trial. Arch Pediatr Adolesc Med 166, 248-255.

71. Fiechtner L, Kleinman K, Melly SJ et al. (2016) Effects of proximity to supermarkets on a randomized trial studying interventions for obesity. Am J Public Health 106, 557-562.

72. Goldberg JP, Folta SC, Eliasziw M et al. (2015) Great Taste, Less Waste: a cluster-randomized trial using a communications campaign to improve the quality of foods brought from home to school by elementary school children. Prev Med $\mathbf{7 4}$, 103-110.

73. Heneman K, Junge SK \& Zidenberg-Cherr S (2008) Reading Across My Pyramid, a nutrition and health education curriculum, increases the health behavior knowledge of lower elementary students. J Child Nutr Manag 32. issue 1, https://schoolnutrition.org/5--News-and-Publications/4--TheJournal-of-Child-Nutrition-and-Management/Spring-2008/ Volume-32,-Issue-1,-Spring-2008---Heneman;Junge;ZidenbergCherr/.

74. Baranowski T \& Domel SB (1994) A cognitive model of children's reporting of food intake. Am J Clin Nutr 59, 1 Suppl., 212S-217S

75. Matheson DM, Hanson KA, McDonald TE et al. (2002) Validity of children's food portion estimates. Arch Pediatr Adolesc Med 156, 867-871.

76. Livingstone MB \& Robson PJ (2000) Measurement of dietary intake in children. Proc Nutr Soc 59, 279-293.

77. Livingstone MBE, Robson PJ \& Wallace JMW (2004) Issues in dietary intake assessment of children and adolescents. $\mathrm{Br} \mathrm{J}$ Nutr 92, 213-222.

78. Glen KE, Thomas HM, Loebach JE et al. (2013) Fruit and vegetable consumption among children in a socioeconomically disadvantaged neighbourhood. Can J Diet Pract Res $\mathbf{7 4}$, 114-118.

79. Blinkhorn A, Freeman R \& Oliver M (2009) Commentary on: Do school break-time policies influence child dental health and snacking behaviours? An evaluation of a primary school programme. Br Dent J 206, 616.

80. Grummon AH, Sokol RL, Hecht CA et al. (2018) Assessing Beverage Intake in Children and Adolescents: State of the Science, Recommendations, and Resources for Evaluation. http://npi.ucanr.edu/files/287170.pdf (accessed March 2019). 